\title{
Increasing Effectiveness of Genetically Modifying Mosquito Populations: Risk Assessment of Releasing Blood-Fed Females
}

\author{
Siyang Xia, ${ }^{*} \dagger$ Jonah Ury, $†$ and Jeffrey R. Powell \\ Department of Ecology and Evolutionary Biology, Yale University, New Haven, Connecticut
}

\begin{abstract}
Releasing mosquito refractory to pathogens has been proposed as a means of controlling mosquito-borne diseases. A recent modeling study demonstrated that instead of the conventional male-only releases, adding blood-fed females to the release population could significantly increase the program's efficiency, hastening the decrease in disease transmission competence of the target mosquito population and reducing the duration and costs of the release program. However, releasing female mosquitoes presents a short-term risk of increased disease transmission. To quantify this risk, we constructed a Ross-MacDonald model and an individual-based stochastic model to estimate the increase in disease transmission contributed by the released blood-fed females, using the mosquito Aedes aegypti and the dengue virus as a model system. Under baseline parameter values informed by empirical data, our stochastic models predicted a 1.1-5.5\% increase in dengue transmission during the initial release, depending on the resistance level of released mosquitoes and release size. The basic reproductive number $\left(R_{0}\right)$ increased by $0.45-3.62 \%$. The stochastic simulations were then extended to 10 releases to evaluate the long-term effect. The overall reduction of disease transmission was much greater than the number of potential infections directly contributed by the released females. Releasing blood-fed females with males could also outperform conventional male-only releases when the release strain is sufficiently resistant, and the release size is relatively small. Overall, these results suggested that the long-term benefit of releasing blood-fed females often outweighs the short-term risk.
\end{abstract}

\section{INTRODUCTION}

It has long been a goal of vector biologists to genetically modify mosquitoes and other vectors to make them less capable of transmitting pathogens. ${ }^{1-4}$ In almost all such proposals, releasing males has been the choice as only females take blood meals, and thus only females transmit diseases (although exceptions exist, e.g., the Wolbachia-infected mosquito release in Australia ${ }^{5}$ ). Releasing females raises concerns of increasing disease transmission (if the release strain is not completely refractory to transmission) and increasing biting rates, adding a nuisance factor that may reduce acceptance by the community into which female mosquitoes are released. However, releasing females could also benefit the control program. For example, Robert et al. ${ }^{6}$ showed that bi-sex or female-only releases have higher efficiency at reducing mosquito population density. Recently, Xia et al. ${ }^{7}$ modeled a proposed scheme, first made by Powell and Tabachnick, ${ }^{8}$ and named "genetic shifting," to genetically modify natural mosquito populations by releasing mosquitoes selected in the laboratory to be refractory to transmission using classical artificial selection methods. The capacity of transmitting diseases in mosquitoes is at least partially genetically determined, ${ }^{9,10}$ so releasing refractory mosquitoes could alter the gene pools in the target mosquitoes and "shift" the subsequent generations to be less competent. ${ }^{8}$ Xia et al. ${ }^{7}$ considered several release strategies, including the standard one of releasing only males, but also a scheme to release females that had been blood-fed before being released along with males. This new releasing scheme was shown to greatly increase the efficiency of the genetic modification of the target population. It induced faster genetic modification and reduced the effort and time needed to significantly reduce the vector competence (VC) of the target

*Address correspondence to Siyang Xia, Harvard T.H. Chan School of Public Health, 677 Huntington Ave., Kresge Bldg. 9th Floor, Boston, MA 02115. E-mail: sxia@hsph.harvard.edu

†These authors contributed equally to this work. population. This is due to both doubling the release numbers by including both sexes and pre-fed females being primed to directly contribute on the order of 50-100 eggs to the target population, multiplying the effect of each released female. ${ }^{7}$

Implementing this female-included release strategy likely involves many practical concerns, perhaps the most important is whether the increased efficiency (shortened time to reach a significant reduction in disease transmission capacity of the vectors) outweighs any immediate increase of disease transmission caused by the releases of females? There are good reasons to expect that releasing pre-blood-fed females may have minimal effects on disease transmission. Figure 1 shows a simplified and idealized time line for such releases and the basis of our models. After blood-feeding, female mosquitoes switch from seeking a blood meal to seeking egg-laying sites. ${ }^{11,12}$ When a pre-fed female is released, it takes several days for her to deposit all her eggs, so seeking a second blood meal (potentially infectious) is delayed (Figure 1B). If the second blood meal is infectious, there is another period of extrinsic incubation before she can transmit the pathogen. ${ }^{13-17}$ Because of the relatively high mortality rate of adult mosquitoes in the wild (Figure 1C), the probability of a released female surviving until becoming infectious and actually transmitting disease would be minimal. ${ }^{18-20}$ Because the surviving females have been selected to be largely refractory to pathogens (the reason they are released), impact on disease incidence would be further reduced.

To better inform empirical applications of releasing blood-fed female mosquitoes along with males (referred to as "blood-fed female releases" in the rest of the article, but the release includes males), we quantified the expected increase in disease transmission that would be attributed to the released females. We addressed this question by constructing an individual-based stochastic model and calculating the basic reproductive number $\left(R_{0}\right)$ according to the Ross-MacDonald model. ${ }^{21}$ The parameters in both approaches were guided by empirical studies on the interaction between the dengue virus and the mosquito Aedes aegypti, the major vector of several diseases including 
A

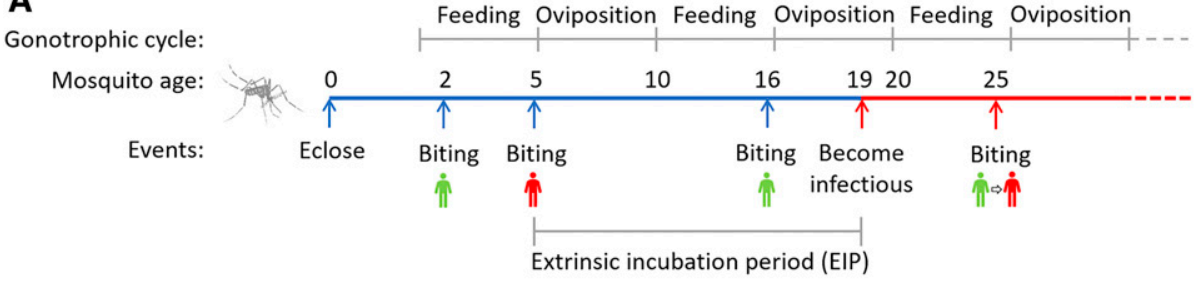

B

Gonotrophic cycle:

Mosquito age:

Events:
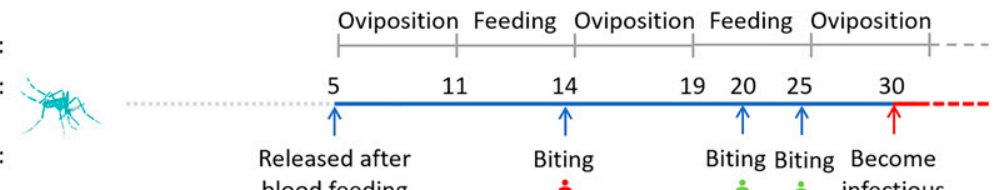

Released after

blood feeding

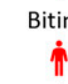

Biting

C

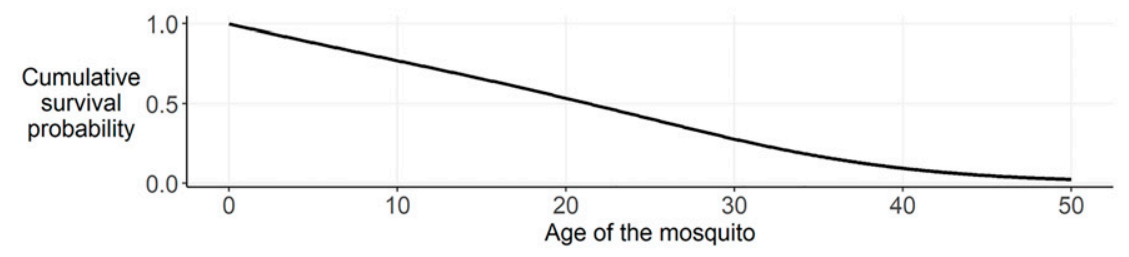

D

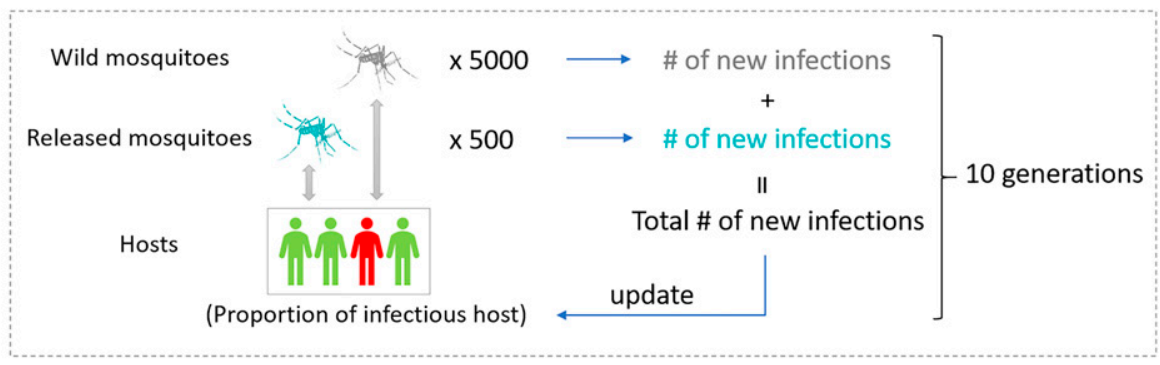

Iterate 100 times

FIGURE 1. Illustration of the individual-based stochastic model. (A and B) Idealized demonstration of the life events, gonotrophic cycle, and the infection status of a wild Aedes aegypti female (A), and a released female after being blood-fed before released (B). The blood-fed released mosquitoes start at the oviposition phase upon being released, instead of the feeding phase. The blue and red lines represent the noninfectious and infectious statuses of the mosquito, and the green and red human icons represent healthy and infectious human hosts, respectively. The timing of all events in both figures for demonstration only and are not constant for all individuals. Because a released female is already blood-fed and needs to finish oviposition before biting, the timing when she becomes infectious is delayed, as represented here by the shorter redline in (B) than that in (A). (C) Probability a mosquito lives until a certain age. The curve was calculated from Equation 6 in the main text. (D) Structure and workflow of the individual-based stochastic model. Each iteration is composed of a simulation of 5,000 wild mosquitoes and a certain number of the released mosquitoes (depending on the release ratio, e.g., 500 when the release ratio is $10 \%$ ) for 10 generations. The total infections in each generation from both the wild and released mosquitoes were used to update the proportion of infectious humans for the next generation. For each parameter combination, 100 iterations were implemented to account for the variations due to model stochasticity. This figure appears in color at www.ajtmh.org.

dengue, yellow fever, chikungunya, and Zika. ${ }^{22}$ We then extended the stochastic model to multiple generations and multiple releases, to evaluate the trade-off between the short-term risk and long-term benefit of releasing pre-blood-fed mosquitoes. Last, uncertainty and sensitivity analyses were used to examine variation in the model outcomes due to uncertainties in parameter estimation, and to identify the relative influence of parameters on the models.

\section{MATERIALS AND METHODS}

The Ross-MacDonald model. We first calculated the basic reproductive number $\left(R_{0}\right)$ for dengue transmission by $A e$. aegypti and explored how the addition of blood-fed females modified it. The basic reproductive number estimates how many human infections in a single disease cycle will result from the first infected human introduced into a completely susceptible human population: How many people "patient zero" will infect? ${ }^{21}$ A large number of studies have modified the model to estimate $R_{0}$ for mosquito-borne diseases ${ }^{23}$; for simplicity and generality, we adopted the most simplified and well-characterized Ross-MacDonald model ${ }^{21,24}$ :

$$
R_{0}=\frac{m a^{2} p^{N} b}{-\ln (p)} \times \frac{c}{r},
$$

where $m$ is the ratio of mosquito to human population density, $a$ is the mosquito's biting rate, $p$ is the daily survival probability, 
$N$ is the extrinsic incubation period (EIP, the duration for the virus to amplify in the mosquito and become ready to infect another host), $b$ is the mosquito VC (the ability of a mosquito to get infected and transit to another host), $c$ is the probability of a mosquito getting infected when biting an infectious human, and $r$ is the recovery rate of human patient. ${ }^{21,25}$ At least two bites are necessary for a mosquito to transmit the pathogen (one to acquire the pathogen and the other one to transmit it to a new host), so the biting rate is squared in Equation 1. In addition, the mosquito needs to survive at least the EIP, the probability of which is $p^{N}$. Our $R_{0}$ calculations assume that mosquitoes exclusively feed on humans.

We modified Equation 1 to incorporate the addition of the released mosquitoes. We assumed the same human infectious rate $(c)$ and recovery rate $(r)$, and that wild and released mosquitoes exhibit the same daily survival rates $(p)$, daily biting rate $(a)$, and EIP $(N)$. Because the mosquitoes have a lower VC and released in quantities normally smaller than the wild population size, both $m$ and $b$ are expected to be lower for the laboratory-reared mosquitoes. In addition, the blood-fed released mosquitoes must finish laying eggs before they seek a second blood meal, so they have to survive an extra oviposition duration, $O$, in addition to the EIP to be infectious. We also assume no interactions between the wild and the released mosquitoes (e.g., no competition). The $R_{0}$ contributed by both the wild and the released mosquito populations is then calculated as the largest eigenvalue of the nextgeneration matrix26,27 (see Supplemental Material for the derivation):

$$
R_{0}^{*}=\frac{m_{w} a^{2} p^{N} b_{w}}{-\ln (p)} \times \frac{c}{r}+\frac{m_{r} a^{2} p^{N+O} b_{r}}{-\ln (p)} \times \frac{c}{r},
$$

where the subscripts $w$ and $r$ indicate values specific to the wild versus the released mosquitoes. The first and second parts (before and after the addition) describe the contribution of the wild population and the released population, respectively. The parameter values in this model are depicted in Table 1. We then calculated the proportional increase of $R_{0}$ resulting from mosquito release:

$$
\frac{R_{0}^{*}-R_{0}}{R_{0}}=\frac{m_{r} b_{r} p^{O}}{m_{w} b_{w}},
$$

We considered four baseline scenarios, with the released mosquitoes having a VC of 0.2 or 0.05 , and the size of the release being $10 \%$ or $20 \%$ of the size of the wild population (Table 1).

In addition, we also performed a one-way sensitivity analysis, varying each parameter value across a reasonable range informed by empirical studies on dengue and $A e$. aegypti, and calculated the proportional increase of $R_{0}$. Because only six of the ten parameters have an impact (Equation 3), we kept the other four parameters $(a, N, c$, and $r$ ) constant during the sensitivity analysis (Table 1 ). We assumed that a reasonable release program would release $5-50 \%$ mosquitoes of the size of the wild populations, and the released individuals should be more resistant than the wild mosquitoes. We allowed a maximal VC of 0.4 for the released mosquitoes when the wild mosquitoes have a VC of 0.524 , but in reality, the released mosquitoes should be almost completely resistant. The range of the VC for the wild mosquitoes was estimated according to field studies. ${ }^{29,30}$ Last, a blood-fed female mosquito would take around 3 days or longer before it is ready to lay eggs, so we tested at a range of 3 to 7 days.

Individual-based stochastic model. To complement the basic reproductive number calculations and allow for more flexibility and stochasticity, we built an individual-based stochastic model. ${ }^{33}$ The model follows the adult life cycle of a single Ae. aegypti female with a series of stochastic events such as biting, oviposition, death, and transmitting pathogens. It then replicates this individual simulation to form population-wide estimates. We simulated the number of human infections contributed by the wild and released mosquitoes separately, which allows us to quantify the increase in disease transmission resulting from the release program. We first focus on the effect of the initial single release, which likely presents the greatest risk in a mosquito release program, as the beneficial effect (e.g., reduction of VC of the mosquitoes) has not taken effect yet, but the released females has already been added to the disease transmission cycle.

We first simulated the life of a wild mosquito. After an initial two days of maturation, the life cycle of a female mosquito comprised gonotrophic cycles. Each cycle includes a biting phase and an oviposition phase, and the mosquito alternates between them (Figure 1A). The duration of each phase follows a truncated normal distribution (Table 2) and is sampled separately for each gonotrophic cycle. Within the feeding phase, the model samples the number of bites per feeding phase from a Poisson distribution with a mean of two bites per cycle. Each bite can be infectious: the probability of which is the product of the proportion of infectious humans in the human population and the mosquito's VC. Although many definitions of VC exist,

TABLE 1

\begin{tabular}{|c|c|c|c|}
\hline Parameter & Baseline & Range & Reference \\
\hline Mosquito-human ratio of wild mosquitoes $\left(\mathrm{m}_{\mathrm{w}}\right)$ & 4 & $0.8-8$ & 25 \\
\hline Mosquito-human ratio of released mosquitoes $\left(m_{r}\right)$ & 0.4 or $0.8^{*}$ & $0.2-2$ & 7 \\
\hline Daily biting rate $(a)$ & 0.7 & $0.7 \dagger$ & 28 \\
\hline Daily survival rate $(p)$ & 0.83 & $0.8-0.975$ & 18 \\
\hline Extrinsic incubation period $(N)$ & 11 & $11 \dagger$ & $14,17,25$ \\
\hline Duration of oviposition $(O)$ & 4 & $3-7$ & 19 \\
\hline Vector competence of wild mosquitoes $\left(b_{w}\right)$ & 0.524 & $0.25-0.825$ & 29,30 \\
\hline Vector competence of released mosquitoes $\left(b_{r}\right)$ & 0.2 or $0.05^{*}$ & $0-0.4$ & 7 \\
\hline Human infectiousness $(c)$ & 0.5 & $0.5 \dagger$ & 31,32 \\
\hline Human recovery rate $(r)$ & 0.143 & $0.143 \dagger$ & 31,32 \\
\hline
\end{tabular}

Parameter values and ranges used in the Ross-MacDonald model informed by the references provided

baseline scenarios were calculated with

† Keep a, $N, c$, and $r$ constant as they do not affect the proportional increase of $R_{0}$ (Equation 3 ). 
TABLE 2

Parameter values and ranges for the individual-based stochastic model

\begin{tabular}{|c|c|c|c|c|}
\hline Parameter ${ }^{\star}$ & Probability distributiont & Baseline & Range/distribution $\neq$ & Ref \\
\hline Proportion of infectious human & Constant & 0.05 & $0.001-0.05$ & \# \\
\hline Age when released & Constant & 5 & $3-8$ & ** \\
\hline Wild mosquito VC & Normal, truncated at $0-1$ & $\begin{array}{l}\text { Mean: } 0.524 \\
\text { SD: } 0.125\end{array}$ & $\begin{array}{l}0.25-0.825 \\
0.05-0.3\end{array}$ & 29,30 \\
\hline Release mosquito VC & Normal, truncated at $0-1$ & $\begin{array}{l}\text { Mean: } 0.2 \text { or } 0.05 \S \\
\text { SD: } 0.125\end{array}$ & $\begin{array}{l}0-0.4 \\
0.05-0.3\end{array}$ & 7 \\
\hline Duration of oviposition & Normal, truncated to $>3$ & $\begin{array}{l}\text { Mean: } 4 \\
\text { SD: } 1\end{array}$ & $\begin{array}{l}\text { Truncated normal }(\mu=4, \sigma=2, a=3, b=7) \| \\
\text { Gamma }(k=4, \theta=0.25) \Phi\end{array}$ & 19,34 \\
\hline Duration of feeding & Normal, truncated to $>1$ & $\begin{array}{l}\text { Mean: } 4 \\
\text { SD: } 1\end{array}$ & $\begin{array}{l}\text { Truncated normal }(\mu=4, \sigma=2, a=1, b=7) \\
\text { Gamma }(k=4, \theta=0.25)\end{array}$ & 19,34 \\
\hline Extrinsic incubation period & Normal, truncated to $>5$ & $\begin{array}{l}\text { Mean: } 11 \\
\text { SD: } 1\end{array}$ & $\begin{array}{l}\text { Truncated normal }(\mu=11, \sigma=3, a=5, b=17) \\
\text { Gamma }(k=4, \theta=0.25)\end{array}$ & $14,17,25$ \\
\hline Bites per gonotrophic cycle & Poisson & Mean: 2 & Truncated normal $(\mu=2, \sigma=2, a=1, b=10)$ & $19,30,35$ \\
\hline Release size ratio & Constant & 0.1 or $0.2 \S$ & $0.05-0.5$ & ** \\
\hline Proportion of successful feeding & Constant & 1 & $0.5-1$ & ** \\
\hline Hazard function parameter, $a$ & Constant & 0.0018 & $\begin{array}{l}\text { Truncated normal }(\mu=0.0018, \sigma=0.0009, \\
\quad a=0.0009, b=0.0027)\end{array}$ & 20 \\
\hline Hazard function parameter, $b$ & Constant & 0.1416 & $\begin{array}{l}\text { Truncated normal }(\mu=0.1416, \sigma=0.0454 \text {, } \\
\quad a=0.0962, b=0.1870)\end{array}$ & 20 \\
\hline Hazard function parameter, $s$ & Constant & 1.073 & $\begin{array}{l}\text { Truncated normal }(\mu=1.073, \sigma=0.6404 \text {, } \\
\quad a=0.4579, b=1.6881)\end{array}$ & 20 \\
\hline Hazard function parameter, $c$ & Constant & 0.022 & $\begin{array}{l}\text { Truncated normal }(\mu=0.022, \sigma=0.022 \\
\qquad a=0, b=0.06)\end{array}$ & $\dagger \dagger$ \\
\hline
\end{tabular}

$\mathrm{VC}=$ vector competence

* Bold parameters were included in the sensitivity analysis. The rest of the parameters were included in the uncertainty analysis.

†The distribution used to sample stochastic events or mosquito VC within each simulation.

$\ddagger$ The range for sensitivity analysis or the sampling distribution for uncertainty analysis.

$\S$ The four baseline scenarios result from the combinations of two mean VC of released mosquitoes and two release sizes.

$\|$ Truncated normal distribution: $\mu=$ mean, $\sigma=\mathrm{SD}, a=$ lower bound, $b=$ upper bound.

I Gamma distribution: $k=$ shape, $\theta=$ scale.

\# The parameter varies by the target human community.

${ }^{\star *}$ Variables describing the release program.

$\dagger+W e$ adjusted this variable so that the hazard function matches the wild mosquito survival rate.

we define VC in this study as the probability that a mosquito will become infectious if it bites an infected human. ${ }^{29,30,36-38}$ We assumed the VC follows a normal distribution truncated at 0 and 1 with different means for the wild and the released mosquito population, respectively (Table 2 ). The VC of each mosquito is randomly sampled and remains constant throughout its life. If the mosquito becomes infected after a bite, the model randomly samples the duration of the EIP from a normal distribution, with a mean of 11 days as documented under laboratory conditions. ${ }^{25}$ The mosquito becomes infectious after the incubation period. Every bite after that stands to infect its human host, given the host is not already infected (a probability of randomly encountering a susceptible host, i.e., 1- proportion of infectious hosts). When an infected mosquito bites a noninfected human, we assume a $100 \%$ chance of transmission for simplicity reasons. Relaxing this assumption should affect the wild and the released mosquitoes similarly, so we do not think it would affect our results.

In addition to gonotrophic cycles and infection status, we randomly sampled each mosquito's life span in our model, following an empirically estimated survival function. Mosquitoes likely exhibit a nonlinear survival curve. ${ }^{18-20}$ Styer et al. ${ }^{20}$ demonstrated that a logistic Makeham hazard function best matches the observed survival of laboratory-reared blood-fed mosquitoes:

$$
U_{x}=\frac{a e^{b x}}{1+\left(\frac{a s}{c}\right)\left(e^{b x}-1\right)}+c,
$$

where $U_{x}$ is the hazard rate, $a$ is the initial mortality rate, $b$ is the exponential mortality increase with age, $x$ is the age, $s$ is the degree of mortality deceleration, and $c$ is the age-independent hazard rate. We used the mean value of $a, b$, and $s$ reported in Styer et al. ${ }^{20}$ in our baseline scenario. We increased the ageindependent hazard rate, $c$, to decrease the median survival to 21 days (Harrington L., personal communication). This assumption better matches mosquito survival in the field; increasing the age-independent mortality parameter tracks the way environmental factors such as predation and extreme weather present an age-independent risk to mosquito survival. Using a time step of 0.1 days, we calculated the probability of the mosquito surviving from day $t$ to day $t+0.1$ as:

$$
p_{t \rightarrow t+0.1}=e^{-U_{x} \times 0.1}
$$

The cumulative probability of the mosquito surviving till age $T$ is then calculated as:

$$
P(T)=\prod_{t=0}^{t=T-0.1} p_{t \rightarrow t+0.1}
$$

Equation 6 is displayed in Figure 1C. For each mosquito in the model, we randomly sample its age of death according to this cumulative survival probability. We then simulate multiple gonotrophic cycles of the mosquito as described earlier until she dies, and summarize the number of infectious bites contributed by the mosquito.

Using this model framework, we first simulated the disease contributed by 5,000 wild mosquitoes and then added the released mosquitoes. The release ratio is expressed as a proportion of the wild mosquito population size (e.g., 10\% represents releasing 500 mosquitoes) in the baseline 
scenarios. We assume the released mosquitoes are 5 days old when starting the simulation (i.e., time spent in the laboratory/ facility before being released), and that they have a lower VC (Table 2). ${ }^{7}$ Blood-fed mosquito females enter the simulation at the beginning of the oviposition phase, in contrast to the nonfed and wild mosquitoes which begin in the biting phase (Figure 1B). Although we recognize that Ae. aegypti females often take multiple blood meals in any single gonotrophic cycle, we assume this will be minimized if the released females are allowed to feed ad libidum on a blood source before release, so they are fully fed. It is still possible that some non-fed females may be accidentally released. Therefore, we considered different ratios of successful feeding in our simulation (100\% in the baseline scenarios) to assess the risk of this practical limitation. We assumed that the release mosquitoes share the same parameter values as the wild mosquitoes for the rest of the parameters (e.g., duration of oviposition, EIP, etc.; Table 2) and have the same age-dependent survival curve, except that they suffer no mortality before being released.

After simulating all mosquitoes from the wild population and the released population, we calculated the proportional increase of disease due to the release. This proportion was calculated as the total number of dengue incidence transmitted by the released mosquitoes divided by that transmitted by the wild mosquitoes. Last, we iterated the entire simulation 100 times to account for the stochasticity, generating an empirical distribution of the proportional increase of disease transmission. Increasing the number of iterations did not reduce stochasticity or affect the model results.

Extending to multiple releases. Proposed release programs likely last for more than just one release. To assess the effectiveness and risk of releasing blood-fed females multiple times, we extended the aforementioned simulation to 10 releases, assuming one release per mosquito generation and nonoverlapping generations. Specifically, we iterated our simulation (5,000 wild and 500 released females at baseline) 10 times. For each successive generation, we first updated the VC of the wild (target) mosquito population according to the release-induced genetic shift. This was calculated using the genetic shifting model described in Xia et al. $^{7}$ (see Supplemental Figure S1 for an example). The two models (genetic shifting model and the current one) share five parameters: VC mean and SD in the initial wild population and the released population, and the size of each release. For the rest of the parameters unique to the genetic shift model, we used the baseline value described in Table 1 in Xia et al. ${ }^{7}$ The outcome of the genetic shifting model is the mean VC of the wild mosquito population across the 10 generations, which were used in this study for the simulation of each of the 10 releases. The proportion of successful feeding before releasing was not incorporated in the genetic shift model (the model in Xia et al. ${ }^{7}$ always assumed perfect prerelease feeding). To account for the effect of this parameter, we assumed that VC changed linearly between scenarios of $100 \%$ feeding (i.e., releasing blood-fed females) and $0 \%$ feeding (i.e., releasing both sexes without feeding; see the following paragraphs for more details). We then adjusted the VC in the 10 generations according to the feeding ratio when it is not $100 \%$.

In addition to updating VC, we adjusted the proportion of infectious humans for each release. To do that, we assumed the human infectiousness before any releases ( $5 \%$ at baseline,
$\mathrm{HI}_{1}$ ) corresponds to the number of infections contributed by the wild mosquitoes in the first generation $\left(N_{\text {wild, } 1}\right)$. We then calculated the successive generation's human infectiousness proportionally as $\mathrm{HI}_{\mathrm{i}}=N_{\text {total, } i-1} / N_{\text {wild, } 1} \times \mathrm{HI}_{1}$, where $i$ indicates generations and $N_{\text {total, } i-1}$ is the total number of new dengue infection simulated in the last generation. For example, assuming $5 \%$ human infectiousness to start, if the wild mosquitoes infected 100 people in the first generation, and in total 110 people were infected after the first release, the human infectiousness proportion in the second generation would be 5.5\%.

Extending the simulation to multiple releases also allows a comparison between different releasing strategies. We simulated the scheme in which only males are released and a third one in which both sexes were released, but females were not pre-fed in the laboratory. The male-only release did not directly contribute to disease transmission, whereas the "bothsex" releases added females that enter the simulation at the beginning of the feeding phase instead of the oviposition phase, as shown in Figure 1B. The three release strategies also differ in the change of VC across generations, which were calculated as in Xia et al. ${ }^{7}$ Last, we also simulated a "control" scheme with no releases.

For each multigenerational simulation, we calculated the number of dengue infections in each generation as proportional to the number of infections by the wild mosquitoes in the first generation. We separated the total number of infections contributed by the wild and released mosquitoes. Moreover, we summed the number of infections across all 10 generations and calculated the proportion to the total infections in the control scheme. As in the single-generation simulation, we iterated the simulation 100 times to obtain the distribution of the outcome variables.

Uncertainty analysis and sensitivity analysis. In light of previous modeling efforts that examined the effect of parameter uncertainties, ${ }^{39-42}$ we performed uncertainty and sensitivity analyses on the individual-based stochastic model. We categorized the 19 parameters in the stochastic model into two groups (Table 2). The first group comprises variables that describe a mosquito's life events, for instance, the duration of the blood-feeding phase, the number of bites per cycle, and the parameters defining the survival function (Equation 4). These parameters are assumed not to be affected by the mosquito release programs; we also assumed that the released mosquitoes share the same values for these parameters as their wild counterparts. In other words, we assume these are constants due to the biology of the particular mosquito species and disease. For these parameters, we were mainly concerned about how uncertainty in parameter estimation might contribute to the uncertainty of our simulation results. Therefore, we implemented an uncertainty analysis for these 11 parameters. We randomly sampled 1,000 combinations of these parameters using Latin hypercube sampling ${ }^{43}$ in $R^{44}$ from their reasonable distributions (Table 2), allowing all parameters to vary simultaneously. We ran the multiplegenerational model for each combination and calculated the mean of the 100 iterations, and then summarized the variations of these 1,000 means.

The other group of parameters contains those that describe the wild mosquito population (e.g., VC mean and SD), the host population (proportion of infectious humans), and the release program (mean and SD of the VC of the released mosquitoes, release size, feeding success before releases, and the age of 
mosquitoes at release). These variables are controllable by directly adjusting the release program or choosing the appropriate target population. To examine how each of these parameters influences the model output, we performed sensitivity analyses on all parameters. We first applied one-way sensitivity analysis, in which we varied each parameter individually across its range while keeping other parameters the same as in the baseline scenario. The ranges for each parameter are depicted in Table 2. As described earlier, we calculated the mean model output from the 100 iterations for each parameter value. In addition to the one-way sensitivity, we also implemented a global sensitivity analysis where all parameters vary simultaneously. This allows us to evaluate the effect of each parameter, while the other parameters are also changing. We again used Latin hypercube sampling to randomly sample 1,000 parameter sets, using the same parameter value ranges as in Table 2 . In all sensitivity analyses, the values of the other group of parameters ("uncertainty" parameters) were kept constant as in the baseline (Table 2).

We modeled all three release strategies for each parameter combination in uncertainty and sensitivity analyses: releasing blood-fed females with males, releasing only males, and releasing both sexes without feeding. This provided us an opportunity to directly compare different strategies under a variety of parameter settings. We also used a random forest $(R F)^{45}$ model to evaluate the most influential parameters in determining the best release strategy. The RF model used results from the global sensitivity analysis, with the eight "sensitivity" parameters as the predictor variables. The dependent variable is a categorical variable indicating which release strategy elicits the lowest total number of infections over all generations (i.e., the highest efficiency in reducing disease transmission). Implementing this RF model produces a rank of all parameters, from the most influential to the least.

Scripts for the individual-based stochastic simulation and further analysis were deposited in GitHub (https://github.com/ siyangxia419/Risk-assessment-of-releasing-blood-fedfemales).

\section{RESULTS}

The Ross-MacDonald model. Using the baseline parameter values, the Ross-MacDonald model estimated that $R_{0}$ is 2.482 before any releases (i.e., only the wild mosquitoes). This $R_{0}$ estimation is consistent with studies that estimated $R_{0}$ during dengue outbreaks in the range of 1.24-4.22. ${ }^{46-49} \mathrm{Re}-$ leasing mosquitoes with a VC of 0.2 at $10 \%$ or $20 \%$ of the wild population size boosted $R_{0}$ to 2.526 or 2.572 , respectively, which is a $1.81 \%$ or $3.62 \%$ increase due to the mosquito release. When the released mosquitoes have an even lower VC (0.05), $R_{0}$ became 2.493 (0.45\% increase) and $2.504(0.91 \%$ increase) with $10 \%$ and $20 \%$ release sizes, respectively.

The sensitivity analysis indicated that six variables affect this proportional increase in $R_{0}$ (Supplemental Figure S1): the mosquito-human ratio of the wild and released mosquitoes $\left(m_{w}\right.$ and $\left.m_{r}\right)$, the VC of the wild and released mosquitoes $\left(b_{w}\right.$ and $b_{r}$ ), the duration of oviposition $(O)$, and the daily survival rate $(p)$. Increasing $m_{w}, b_{w}$, and $O$, or decreasing $m_{r}, b_{r}$, and $p$ resulted in smaller increases of $R_{0}$. These results are consistent with our expectations. Releasing more resistant mosquitoes in smaller numbers reduced the risk of increasing disease transmission. As the risk was evaluated as a proportion of the initial $R_{0}$, factors that resulted in larger $R_{0}$ for the wild population (such as large wild mosquito population size and more competent wild mosquitoes) also lessened the proportional risk of releasing. Last, if the mosquitoes require a longer time for oviposition and a lower daily survival rate, the released mosquitoes were more likely to die before transmitting diseases.b

Individual-based stochastic model: baseline. Focusing on the initial generation of release, under the four baseline scenarios, the individual-based model predicted a $1.1-5.5 \%$ increase in dengue transmission from releasing blood-fed female mosquitoes (Figure 2). Releasing larger numbers of mosquitoes ( $20 \%$ versus $10 \%$ of the wild population size) with a relatively high VC $(0.2$ versus 0.05$)$ resulted in a larger disease increase.

Extending the simulation to 10 generations, we observed a rapid decline of new dengue infections when releasing bloodfed females (Figure 3). A temporary increase in disease transmission occurred only in the first two or three generations of releases. Over all 10 generations, releasing mosquitoes with a mean VC of 0.2 or 0.05 and at $10 \%$ or $20 \%$ of the wild population size lowered the total dengue infection to $59.4 \%$ (95\% Cl: 48.5-70.2\%), 46.2\% (39.0-54.1\%), $48.6 \%$ (42.1-56.6\%), and $34.7 \%$ (30.8-39.2\%), respectively, relative to the control scenario of no releases. In comparison, the proportion of dengue cases directly contributed by the released mosquitoes were $1.9 \%$ (1.5-2.5\%), 0.6\% (0.4-0.9\%), $3.3 \%(2.6-4.1 \%)$, and $1.0 \%(0.7-1.3 \%)$, respectively. When comparing it with the two other release strategies (i.e., releasing both sexes without feeding or releasing only males), releasing blood-fed females consistently performed better, resulting in the lowest dengue transmission from the third generation on (Figure 3). This suggests that the long-term benefit of releasing blood-fed females outweighs the shortterm surge of diseases at the initial stages of such a mosquito release program.

Individual-based stochastic model: uncertainty and sensitivity analyses. The ranges of uncertainty in dengue infection from varying the 11 uncertainty parameters are summarized in Figure 4. In general, the effect of parameter uncertainties was amplified in later generations. Releasing blood-fed females still led to the largest reduction in disease transmission after the third release. This result was consistent with what we observed in the baseline scenario (Figure 3A). The total infections across the 10 generations when releasing blood-fed females were, on average, 60.4\% (95\% Cl: $55.3-65.7 \%)$ of that when no releases were conducted. Among them, 1.9\% (1.4-2.6\%) were contributed by the released mosquito. When focusing on each of the 11 parameters, most did not have a strong effect on the simulation outcomes (i.e., total infections relative to the no-release scenario; Supplemental Figure S2). Increasing the number of bites per gonotrophic cycle seemed to diminish the efficiency of the releases, that is, resulted in a higher number of infections, but it affected all release strategies similarly (Supplemental Figure S2E).

For the other eight parameters, one-way sensitivity analysis revealed that the releasing program reduced more disease transmission (i.e., had better performance) over 10 generations (i.e., releases) when 1) a smaller proportion of the target population is infectious (Figure 5A), 2) releasing mosquitoes at older ages (Figure 5B), 3) releasing more refractory mosquitoes into more susceptible wild 


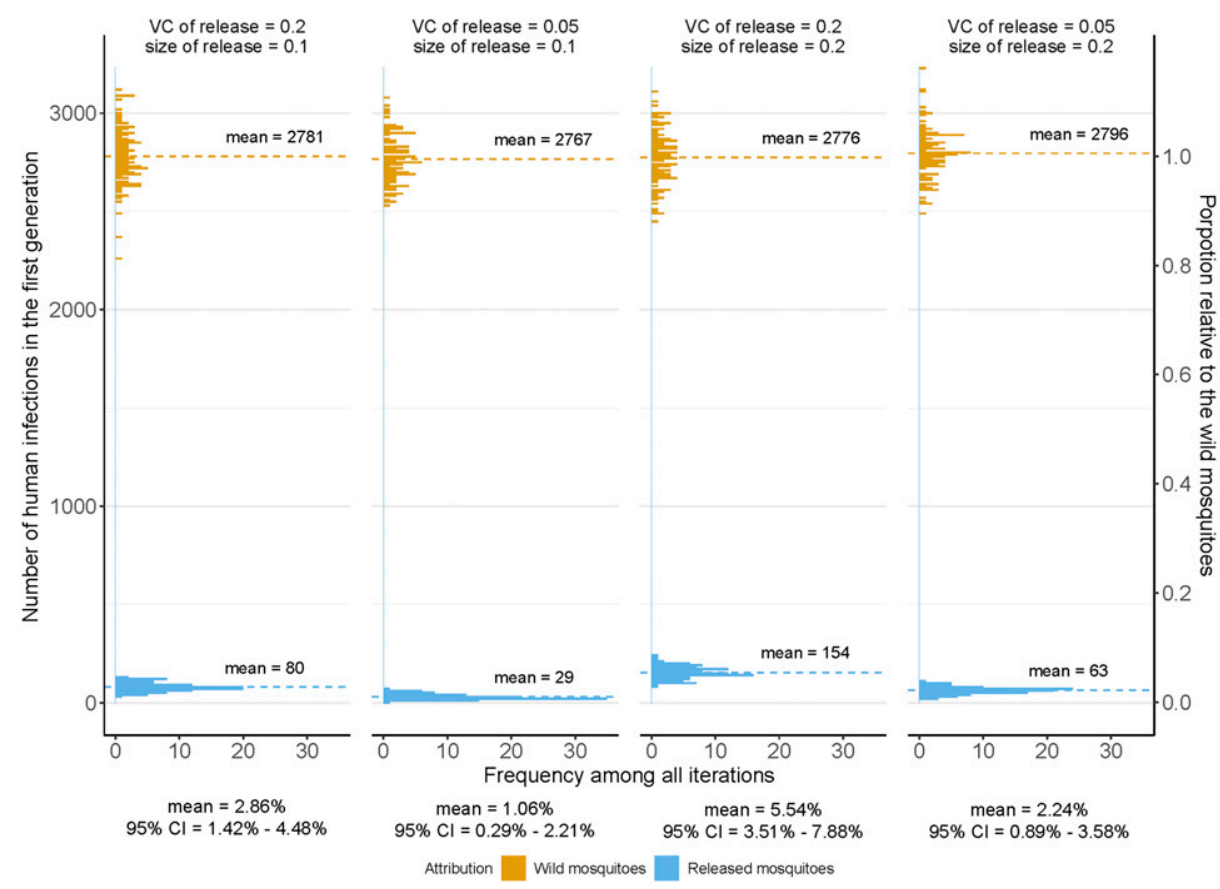

FIGURE 2. Histogram of the number of infections contributed by the wild mosquitoes (brown) and the released blood-fed mosquitoes (blue) during the initial release. The four panels represent the four baseline scenarios with different VCs of the released mosquitoes and different release sizes, which are labeled on top of the panels. We transformed the raw number of incidences (the left $y$-axis) to a proportion relative to the mean wildmosquito-caused infection counts in the first scenario (i.e., 2,781), shown in the second $y$-axis on the right. The means and $95 \%$ Cls of the proportional increase in dengue transmission from the released mosquitoes were indicated at the bottom of each panel. This figure appears in color at www.ajtmh.org.

populations (Figure $5 \mathrm{C}$ and $\mathrm{E})$, 4) releasing larger numbers of individuals each time (Figure 5G), and 5) a higher proportion of the released females successfully fed before being released (Figure $5 \mathrm{H}$, green line). The VC variance within each simulation for both the released and wild mosquito populations has a minimal effect on the model results (Figure 5D and F). The number of infections contributed by the released mosquito alone is shown in Supplemental Figure S3. As expected, releasing less-resistant mosquitoes (relative to the wild mosquitoes) in a larger quantity increased the number of dengue cases transmitted by the released population.

In most parameter settings within the one-way sensitivity analysis, blood-fed female releases outperformed the other two release strategies. However, as the differences in the VC between wild mosquitoes and released mosquitoes decrease, the conventional male-only release becomes the better option. Releasing a larger number of mosquitoes each time (roughly larger than 0.35 the size of the wild mosquito population) also led the blood-fed female release to perform worse than the equivalent male-only release. It is likely that with these parameter settings, releasing blood-fed females did not significantly improve the reduction of VC in comparison with male-only releases. Instead, these released females directly contributed to the large number of infections, offsetting any benefits from the faster VC decline.

Last, we allowed all eight sensitivity parameters to vary simultaneously. In contrast to the observation of the one-way sensitivity analysis (Figure 5), releasing only males resulted in an apparent lower mean of total infections than releasing blood-fed females (Supplemental Figure S4). When taking a closer look at each parameter value combination, we found that for about half of all combinations, releasing blood-fed females was still the better option, whereas in the other half, releasing only males produced the lowest total number of infections. The RF analysis revealed that the most influential factors determining which release strategy is optimal were the mean VC of the released versus the wild mosquitoes, the release size, and the proportion of successful feeding before releasing (Figure 6). Releasing more resistant mosquitoes at a smaller amount each time and ensuring more females were fully fed favored the blood-fed female release strategy (Supplemental Figure S5). This finding is consistent with our one-way sensitivity analysis (Figure 5, comparing the green and the blue lines).

\section{DISCUSSION}

Our initial results indicated that although releasing bloodfed female mosquitoes with males in a genetic modification program may present some short-term risk of increasing diseases, the risk is likely relatively small. The RossMacDonald model and the individual-based model generally agreed on the proportional increase of disease transmission during the initial release. More importantly, our multigenerational models suggested that the long-term benefit of releasing blood-fed females, in terms of reducing total infections over multiple generations, significantly exceeds the shortterm risk. Releasing blood-fed females rapidly decreased the VC of the target populations (e.g., almost two times faster than male-only releases as predicted in Xia et al. ${ }^{7}$ ), which averted a large number of infections in later generations, offsetting the 

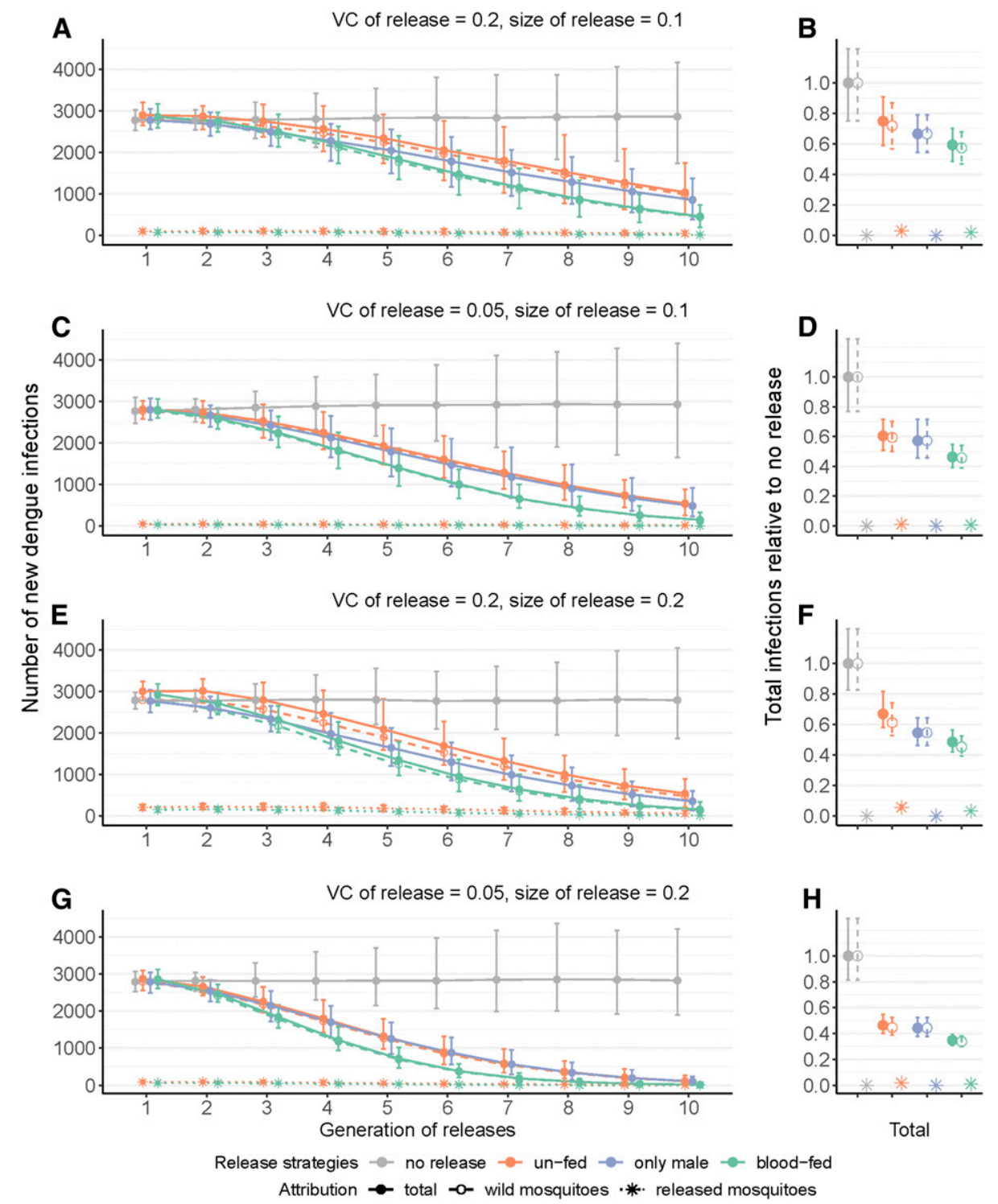

FIGURE 3. The number of dengue infections simulated by the multigenerational model under the four baseline scenarios (indicated at the top of each row of the panel). The four panels on the left $(\mathbf{A}, \mathbf{C}, \mathbf{E}$, and $\mathbf{G})$ summarize the disease incidence per generation, whereas the four panels on the $\operatorname{right}(\mathbf{B}, \mathbf{D}, \mathbf{F}$, and $\mathbf{H})$ show the total infections across all 10 generations, expressed as a proportion of the no-release control scheme. The points and the error bars show the mean and $95 \% \mathrm{Cl}$, respectively. Colors represent release strategies, and the point/line shapes indicate the mosquito groups (total, wild, or released) that contributed to the infection. The gap between the solid lines and the corresponding dashed line thus demonstrated the disease transmitted directly by the released mosquitoes, which was also shown by the star and the dashed lines. This figure appears in color at www.ajtmh.org.

temporary increase of infections. As a result, these releasing strategies could outperform the conventional male-only releases in the long run.

Although our model depends on many parameters describing the gonotrophic cycles and specific human-biting events of $A$ e. aegypti, the uncertainty analysis suggested that varying these parameters does not significantly affect our main findings. Among other parameters, the two most influential model inputs were the size of the release population and the VC of the released females relative to that of the wild individuals. Increasing the size of releases again presented a trade-off: a larger release results in a quicker reduction in VC, thus reducing the time to decrease or stop disease transmission, ${ }^{7}$ but at the same time, it increased the number of infections transmitted directly by the released individuals.
This was observed in both the Ross-MacDonald analysis (Supplemental Figure S1) and the stochastic model (Supplemental Figure S3): release size greater than 20-30\% could lead to a more than $5 \%$ increase in $R_{0}$ or simulated dengue cases. As a result, releasing blood-fed females became less desirable than male-only releases when releasing in large numbers.

On the other hand, reducing VC of the released mosquitoes was always beneficial. Ideally, the released population would have a VC of zero, and thus the released females would not affect disease transmission. However, we considered an imperfectly refractory strain release, which may be more realistic. To obtain approval from relevant authorities, it is unlikely any release would be performed with a refractory strain with a VC greater than $10 \%$. Also, releases would have relatively higher 

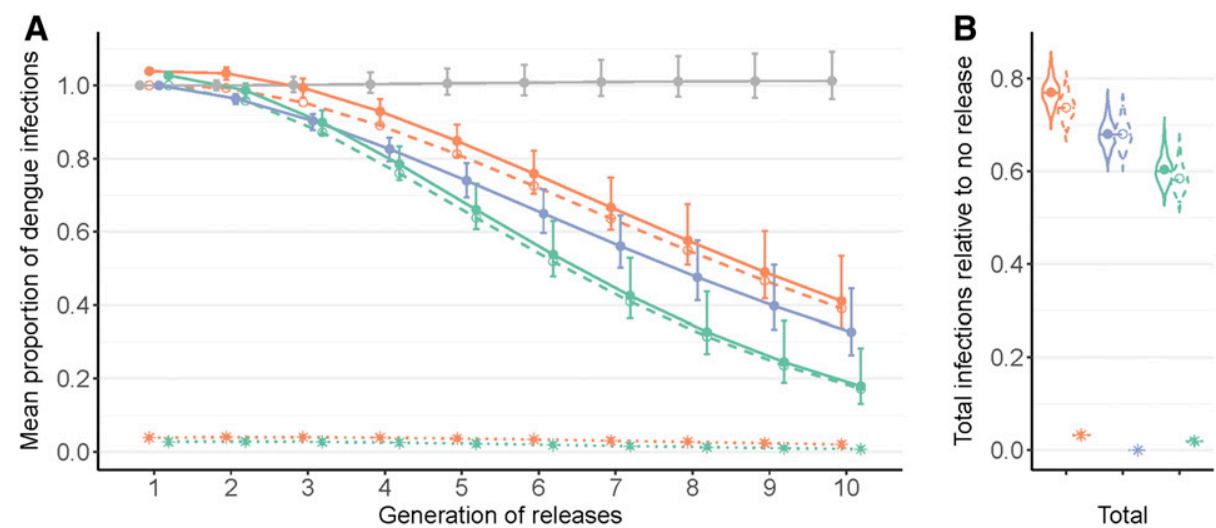

Release strategies $\rightarrow$ no release $\rightarrow$ un-fed $\rightarrow$ only male $\rightarrow$ blood-fed

Attribution $\rightarrow$ total -0 - wild mosquitoes $\cdot *$. released mosquitoes

FIGURE 4. Variations of the simulation results due to uncertainties of 11 parameters (Table 2). (A) Mean and $95 \% \mathrm{Cl}$ of the mean number of dengue infections per generation (mean of the 100 iterations), proportional to the no-release control scheme (gray points and lines) at the first generation. (B) Violin plot showing the distribution of the total infections relative to the no-release control scheme. The proportion was calculated individually for each of the 1,000 parameter combinations. As described in Figure 3, colors represent release strategies, and the point/line shapes indicate the attribution of the infections to different mosquito groups. This figure appears in color at www.ajtmh.org.

efficiency when the target population has a high VC before the release programs.

We recognize that practical issues may arise in releasing blood-fed females. Perhaps, the most important is to be certain that any such program would release only fully blood-fed females. Releasing non-blood-fed females decreased their effectiveness, as they would not be primed to start laying eggs immediately and likely would not lay as many eggs. More importantly, females not taking a blood meal or even partially blood-fed may start biting immediately after being released, thus reducing the time before an infective bite might occur. Indeed, our models predicted that a lower proportion of successful feeding results in more disease transmission by the released mosquitoes, and, over multiple generations,
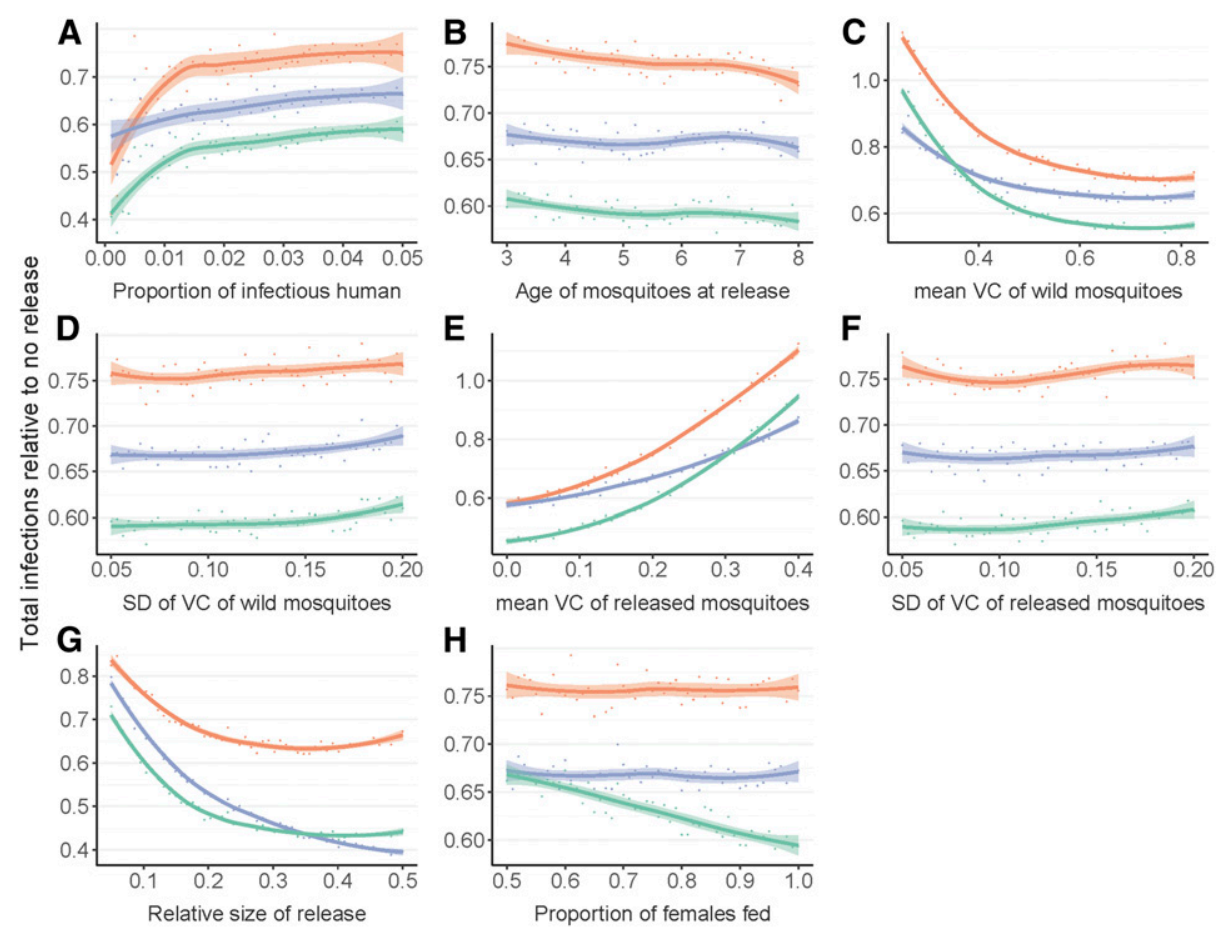

Release strategies $=$ un-fed $=$ only male - blood-fed

FIGURE 5. One-way sensitivity analysis showing the effect of each parameter on the mean total number of infections over 10 generations. The parameters of interest are labeled at the bottom of each panel. The infection numbers were expressed as proportional to the no-release control scheme. Colors represent release strategies. Each point represents a simulation with a different value of the target parameter. The lines and the shades $(95 \% \mathrm{Cl})$ were trend lines estimated using the "stat_smooth" function in ggplot $2^{61}$ in R. Note: In panel $(\mathbf{F})$, the parameter of interest, that is, the proportion of released females successfully fed, only matters when releasing blood-fed females, but the other two releasing strategies were estimated to facilitate comparisons. This figure appears in color at www.ajtmh.org. 


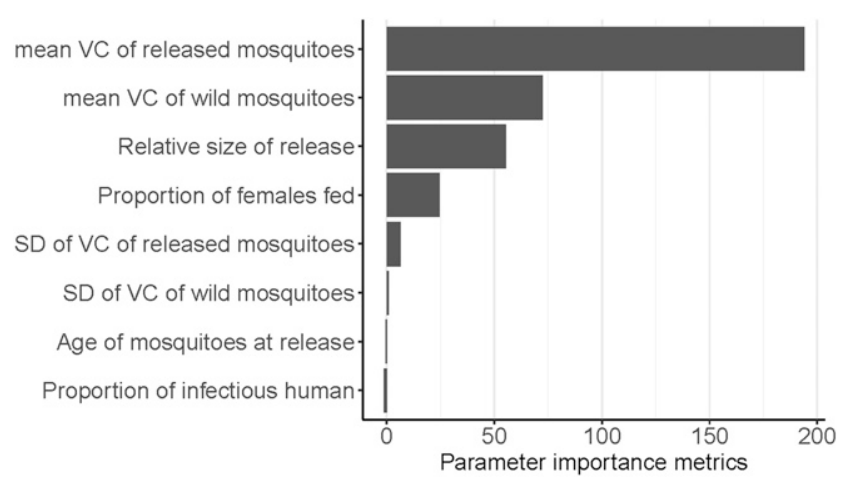

FIGURE 6. Parameter importance in determining the optimal release strategy. The optimal release strategy (e.g., releasing blood-fed females) was determined as that which resulted in the minimal number of total infections in all generations. Parameter importance was generated using the data from the global sensitivity analysis and the random forest model.

diminished the effectiveness in decreasing disease transmission (Supplemental Figure S3). This effect would be more prominent in the initial releases. Therefore, in reality, a carefully implemented release program should ensure that almost all released females are successfully fed, for example, by allowing females to feed multiple times and developing new techniques to separate engorged females.

Our models focused on the life cycle of mosquitoes, assuming a constant environment. Environmental variations, such as temperature and humidity, have been shown to impact EIP and survival. ${ }^{15,17,50}$ However, these environmental conditions likely affect both wild and released mosquitoes similarly, so this assumption should not greatly distort our estimation of the proportional increase of disease transmission. Incorporating these effects could provide a more accurate prediction of disease dynamics. Another potential avenue for future work is to examine the effect of spatial heterogeneity. Many previous studies have suggested that the spatial structure of the mosquito and the host population could affect the prediction of dengue transmission as well as the performance of mosquito control programs. ${ }^{42,46,51-53}$ To incorporate this new dimension, a spatially explicit model framework (e.g., Skeeter Buster ${ }^{52,54,55}$ ) should be used. For instance, using Skeeter Buster, Legros et al. ${ }^{42}$ showed that heterogeneity in the mosquito population could diminish the outcome of releasing transgenic mosquitoes, and compared homogeneous versus point-source release protocols. Other model frameworks could further validate the results from our study and provide additional insight. For example, existing entomological models could be linked to dengue epidemiological models (e.g., SEIR models ${ }^{56-58}$ ). Exploration using different variants of the Ross-MacDonald model could also be helpful. ${ }^{21,23}$ Last, for designing realistic release programs specific to a geographic location, it is also crucial to construct more detailed models that incorporate local data (e.g., detailed human movement pattern and mosquito ecology, etc.) to more accurately predict and optimize the release program, such as the study by Hladish et al. ${ }^{59}$ and study by Legros et al. ${ }^{42}$

Of course, assessing the effects of releasing blood-fed females on disease incidence alone is only one issue of concerns related to this unconventional mosquito release scheme. Even though the temporary addition of disease transmission from these released mosquitoes may not be substantial, it is still an increase in public health risk. Also, the released females will increase the number of bites, which could be a nuisance by themselves. Depending on the local disease burden, local policies, and the public's attitude toward mosquitoes and mosquito releases, this new releasing strategy may not be universally accepted even after proving its high efficacy. More rigorous risk assessment analyses that incorporate additional factors, such as the capacity of the healthcare system, should be conducted. Public education could be important in reducing the communities' resistance toward releasing female mosquitoes. Regulations and surveillance systems should also be in place. Many existing guidelines (e.g., the Arthropod Containment Guidelines ${ }^{60}$ ) could include guidelines specific for female release. Other practical issues surrounding the releasing process should also be carefully considered. For instance, what is the best approach to release the blood-fed mosquitoes, as they might be reluctant to fly? A potentially promising alternative to releasing females, or adult mosquitoes in general, is to release eggs, which has been explored in a few studies. ${ }^{42}$ But how would that compare with releasing blood-fed females? These questions remain open.

To summarize our findings, releasing blood-fed female mosquitoes greatly increases the efficiency and speed to achieve a significant reduction in disease transmission in a genetic modification program while producing a minimal temporary increase in disease incidence. For the best estimates of parameters for Ae. aegypti and dengue that we used, the important recommendations would be 1) the released strain should have as low a VC as achievable and no more than $10 \%$ (Figure 5E); 2) the numbers released in each generation should be as large as practical to achieve a faster reduction in disease transmission ${ }^{5}$; however, if exceeding $30 \%$ the size of the target population, there may be a small additional increase in disease incidents relative to male-only releases (Figure 5G); and 3 ) the proportion of fully blood-fed females should be as high as possible. Otherwise, varying other controllable parameters had minimal effects on the outcome of the release program.

We note that our findings are specific to the mosquito species (Ae. aegypti) and disease considered (dengue). Caution should be taken when trying to extend the conclusion to other disease-vector systems. However, the framework could be adapted to a large range of situations with careful adjustment to the model inputs. If the release of blood-fed females is considered for genetic modification programs aimed at other diseases and vectors (e.g., Anopheles and malaria), parameters specific to these systems should be investigated, and the model should be adjusted to reflect the specific disease transmission cycle (e.g., multiple hosts, different EIP, and vector survival rates).

Received October 1, 2019. Accepted for publication January 29, 2021. Published online March 29, 2021.

Note: Supplemental materials and figures appear at www.ajtmh.org.

Acknowledgments: We thank Virginia Pitzer for her advice and input on the development of the Ross-MacDonald model. We also thank Laura Harrington for the valuable conversation and suggestions on the model parameters. We are grateful to Linda Styer and Thomas Scott for sharing their data on the hazard function of Aedes aegypti. In addition, we thank Yale Center for Research Computing for the technical support. Last, we appreciate all the helpful comments and input from the reviewers and editors that helped us improve this work. 
Financial support: This work was partly supported by the National Institute of Health (Grant UO1-Al115595)

Authors' addresses: Siyang Xia, Jonah Ury, and Jeffrey R. Powell, Department of Ecology and Evolutionary Biology, Yale University, New Haven, CT, E-mails: sxia@hsph.harvard.edu, jonahsury@ gmail.com, and jeffrey.powell@yale.edu.

This is an open-access article distributed under the terms of the Creative Commons Attribution (CC-BY) License, which permits unrestricted use, distribution, and reproduction in any medium, provided the original author and source are credited.

\section{REFERENCES}

1. Beaty BJ, 2000. Genetic manipulation of vectors: a potential novel approach for control of vector-borne diseases. Proc Natl Acad Sci U S A 97: 10295-10297.

2. Beerntsen BT, James AA, Christensen BM, 2000. Genetics of mosquito vector competence. Microbiol Mol Biol Rev 64: 115-137.

3. Collins FH, James AA, 1996. Genetic modification of mosquitoes. Sci Med 3: 52-61.

4. Scott TW, Takken W, Knols BG, Boëte C, 2002. The ecology of genetically modified mosquitoes. Science 298: 117-119.

5. Hoffmann AA et al., 2011. Successful establishment of Wolbachia in Aedes populations to suppress dengue transmission. Nature 476: 454-457.

6. Robert MA, Okamoto KW, Gould F, Lloyd AL, 2014. Antipathogen genes and the replacement of disease-vectoring mosquito populations: a model-based evaluation. Evol Appl 7: 1238-1251.

7. Xia S, Baskett ML, Powell JR, 2019. Quantifying the efficacy of genetic shifting in control of mosquito-borne diseases. Evol Appl 12: 1552-1568.

8. Powell JR, Tabachnick WJ, 2014. Genetic shifting: a novel approach for controlling vector-borne diseases. Trends Parasitol 30: 282-288.

9. Bennett KE, Flick D, Fleming KH, Jochim R, Beaty BJ, Black WCt, 2005. Quantitative trait loci that control dengue-2 virus dissemination in the mosquito Aedes aegypti. Genetics 170: 185-194.

10. Bosio CF, Fulton RE, Salasek ML, Beaty BJ, Black WC, 2000. Quantitative trait loci that control vector competence for dengue-2 virus in the mosquito Aedes aegypti. Genetics 156: 687-698.

11. Farjana T, Tuno N, 2013. Multiple blood feeding and host-seeking behavior in Aedes aegypti and Aedes albopictus (Diptera: culicidae). J Med Entomol 50: 838-846.

12. Klowden MJ, 1981. Initiation and termination of host-seeking inhibition in Aedes aegypti during oöcyte maturation. $J$ Insect Physiol 27: 799-803.

13. Chan M, Johansson MA, 2012. The incubation periods of dengue viruses. PloS One 7: e50972.

14. Nishiura $H$, Halstead SB, 2007. Natural history of dengue virus (denv) -1 and denv -4 infections: reanalysis of classic studies. $J$ Infect Dis 195: 1007-1013.

15. Rohani A, Wong Y, Zamre I, Lee H, Zurainee M, 2009. The effect of extrinsic incubation temperature on development of dengue serotype 2 and 4 viruses in Aedes aegypti (L.). Southeast Asian $J$ Trop Med Public Health 40: 942-950.

16. Tjaden NB, Thomas SM, Fischer D, Beierkuhnlein C, 2013. Extrinsic incubation period of dengue: knowledge, backlog, and applications of temperature dependence. PLoS Negl Trop Dis 7: e2207.

17. Watts DM, Burke DS, Harrison BA, Whitmire RE, Nisalak A, 1987. Effect of temperature on the vector efficiency of Aedes aegypti for dengue 2 virus. Am J Trop Med Hyg 36: 143-152.

18. Maciel-De-Freitas R, Codeco CT, Lourenco-De-Oliveira R, 2007. Daily survival rates and dispersal of Aedes aegypti females in Rio de Janeiro, Brazil. Am J Trop Med Hyg 76: 659-665.

19. Reiter P, 2007. Oviposition, dispersal, and survival in Aedes aegypti: implications for the efficacy of control strategies. Vector-Borne Zoonotic Dis 7: 261-273.
20. Styer LM, Carey JR, Wang J-L, Scott TW, 2007. Mosquitoes do senesce: departure from the paradigm of constant mortality. Am J Trop Med Hyg 76: 111-117.

21. Smith DL, Battle KE, Hay SI, Barker CM, Scott TW, McKenzie FE, 2012. Ross, Macdonald, and a theory for the dynamics and control of mosquito-transmitted pathogens. PLoS Pathogens 8: e1002588.

22. World Health Organization, 2014. A Global Brief on Vector-Borne Diseases. Geneva, Switzerland: WHO.

23. Reiner RC, Jr., Perkins TA, Barker CM, Niu T, Chaves LF, Ellis AM, George DB, Le Menach A, Pulliam JR, Bisanzio D, 2013. A systematic review of mathematical models of mosquito-borne pathogen transmission: 1970-2010. J $R$ Soc Interf 10: 20120921.

24. Macdonald G, 1957. The Epidemiology and Control of Malaria. London, NY, Toronto: Oxford University Press.

25. Armstrong PM, Ehrlich H, Bransfield A, Warren JL, Pitzer VE, Brackney DE, 2018. Successive bloodmeals enhance virus dissemination within mosquitoes and increase transmission potential. BioRxiv. 246306.

26. Diekmann O, Heesterbeek JAP, Metz JAJ, 1990. On the definition and the computation of the basic reproduction ratio $\mathrm{RO}$ in models for infectious diseases in heterogeneous populations. $J$ Math Biol 28: 365-382.

27. Vynnycky E, White R, 2010. An Introduction to Infectious Disease Modelling. Oxford, United Kingdom: Oxford University Press.

28. Scott TW, Amerasinghe PH, Morrison AC, Lorenz LH, Clark GG, Strickman D, Kittayapong P, Edman JD, 2000. Longitudinal studies of Aedes aegypti (Diptera: culicidae) in Thailand and Puerto Rico: blood feeding frequency. $J$ Med Entomol 37: 89-101.

29. Bennett KE, Olson KE, de Lourdes Muñoz M, Fernandez-Salas I, Farfan-Ale JA, Higgs S, Black WC, Beaty BJ, 2002. Variation in vector competence for dengue 2 virus among 24 collections of Aedes aegypti from Mexico and the United States. Am J Trop Med Hyg 67: 85-92.

30. Gonçalves CM, Melo FF, Bezerra JM, Chaves BA, Silva BM, Silva LD, Pessanha JE, Arias JR, Secundino NF, Norris DE, 2014. Distinct variation in vector competence among nine field populations of Aedes aegypti from a Brazilian dengue-endemic risk city. Parasite Vector 7: 320

31. Amaku M, Azevedo F, Burattini MN, Coutinho FAB, Lopez LF, Massad E, 2016. The magnitude and frequency variations of vector-borne infections outbreaks with the Ross-Macdonald model: explaining and predicting outbreaks of dengue fever. Epidemiol Infect 144: 3435-3450.

32. Massad E, Coutinho FAB, Burattini MN, Amaku M, 2010. Estimation of R0 from the initial phase of an outbreak of a vectorborne infection. Trop Med Internat Health 15: 120-126.

33. Gu W, Killeen GF, Mbogo CM, Regens JL, Githure JI, Beier JC, 2003. An individual-based model of Plasmodium falciparum malaria transmission on the coast of Kenya. Trans $R$ Soc Trop Med Hyg 97: 43-50.

34. Christophers SR, 1960. Aedes aegypti (L.) the yellow fever mosquito: its life history, bionomics and structure. Cambridge, UK: Cambridge University Press.

35. Dubrulle M, Mousson L, Moutailler S, Vazeille M, Failloux A-B, 2009. Chikungunya virus and Aedes mosquitoes: saliva is infectious as soon as two days after oral infection. PloS One 4: e5895.

36. Hardy JL, HoukEJ, Kramer LD, Reeves WC, 1983. Intrinsic factors affecting vector competence of mosquitoes for arboviruses. Annu Rev Entomol 28: 229-262.

37. Vega-Rúa A, Zouache K, Girod R, Failloux A-B, Lourenço-deOliveira R, 2014. High level of vector competence of Aedes aegypti and Aedes albopictus from ten American countries as a crucial factor in the spread of Chikungunya virus. $J$ Virol 88: 6294-6306.

38. Souza-Neto JA, Powell JR, Bonizzoni M, 2019. Aedes aegypti vector competence studies: a review. Infect Genet Evol 67: 191-209.

39. Xu C, Legros M, Gould F, Lloyd AL, 2010. Understanding uncertainties in model-based predictions of Aedes aegypti population dynamics. PLoS Negl Trop Dis 4: e830. 
40. Harper EB, Stella JC, Fremier AK, 2011. Global sensitivity analysis for complex ecological models: a case study of riparian cottonwood population dynamics. Ecol Appl 21: 1225-1240.

41. Ellis AM, Garcia AJ, Focks DA, Morrison AC, Scott TW, 2011. Parameterization and sensitivity analysis of a complex simulation model for mosquito population dynamics, dengue transmission, and their control. Am J Trop Med Hyg 85: 257-264.

42. Legros M, Xu C, Okamoto K, Scott TW, Morrison AC, Lloyd AL, Gould F, 2012. Assessing the feasibility of controlling Aedes aegypti with transgenic methods: a model-based evaluation. PLoS One 7: e52235.

43. Carnell R, 2020. Lhs: latin hypercube samples. R Package Version 1.0.2. Available at: https://CRAN.R-project.org/package=lhs.

44. R Development Core Team, 2018. R: A Language and Environment for Statistical Computing. Vienna, Austria: R Foundation for Statistical Computing.

45. Qi Y, 2012. Random Forest for bioinformatics. Ensemble Machine Learning. Boston, MA: Springer, 307-323.

46. Chowell G, Diaz-Duenas P, Miller J, Alcazar-Velazco A, Hyman J, Fenimore P, Castillo-Chavez C, 2007. Estimation of the reproduction number of dengue fever from spatial epidemic data. Math Biosci 208: 571-589.

47. Hsieh YH, Ma S, 2009. Intervention measures, turning point, and reproduction number for dengue, Singapore, 2005. Am J Trop Med Hyg 80: 66-71.

48. Marques C, Forattini O, Massad E, 1994. The basic reproduction number for dengue fever in Sao Paulo state, Brazil: 1990-1991 epidemic. Trans $R$ Soc Trop Med Hyg 88: 58-59.

49. Pinho STRd, Ferreira CP, Esteva L, Barreto FR, Morato e Silva V, Teixeira M, 2010. Modelling the dynamics of dengue real epidemics. Philos Trans $R$ Soc A 368: 5679-5693.

50. Costa EAPdA, Santos EMdM, Correia JC, Albuquerque CMRd, 2010. Impact of small variations in temperature and humidity on the reproductive activity and survival of Aedes aegypti (Diptera, Culicidae). Revista Brasileira de Entomologia 54: 488-493.
51. Chao DL, Longini IM, Jr., Halloran ME, 2013. The effects of vector movement and distribution in a mathematical model of dengue transmission. PloS One 8: e76044.

52. Magori K, Legros M, Puente ME, Focks DA, Scott TW, Lloyd AL, Gould F, 2009. Skeeter Buster: a stochastic, spatially explicit modeling tool for studying Aedes aegypti population replacement and population suppression strategies. PLoS Negl Trop Dis 3: e508.

53. Vanlerberghe V, Gómez-Dantés H, Vazquez-Prokopec G, Alexander N, Manrique-Saide P, Coelho G, Toledo ME, Ocampo CB, Stuyft $\mathrm{PVd}$, 2018. Changing paradigms in Aedes control: considering the spatial heterogeneity of dengue transmission. Revista Panamericana de Salud Pública 41: e16.

54. Okamoto KW, Robert MA, Lloyd AL, Gould F, 2013. A reduce and replace strategy for suppressing vector-borne diseases: insights from a stochastic, spatial model. PLoS One 8: e81860.

55. Legros M, Magori K, Morrison AC, Xu C, Scott TW, Lloyd AL, Gould F, 2011. Evaluation of location-specific predictions by a detailed simulation model of Aedes aegypti populations. PLOS One 6: e22701.

56. Bjørnstad ON, Shea K, Krzywinski M, Altman N, 2020. Modeling infectious epidemics. Nat Methods 17: 455-456.

57. Newton EA, Reiter $P, 1992$. A model of the transmission of dengue fever with an evaluation of the impact of ultra-low volume (ULV) insecticide applications on dengue epidemics. Am J Trop Med Hyg 47: 709-720.

58. Syafruddin S, Noorani M, 2012. SEIR model for transmission of dengue fever in Selangor Malaysia. IJMPS 9: 380-389.

59. Hladish TJ, Pearson CA, Chao DL, Rojas DP, Recchia GL, GómezDantés H, Halloran ME, Pulliam JR, Longini IM, 2016. Projected impact of dengue vaccination in Yucatán, Mexico. PLoS Negl Trop Dis 10: e0004661.

60. American Committee of Medical Entomology American Society of Tropical Medicine and Hygiene, 2019. Arthropod containment guidelines, version 3.2. Vector Borne Zoonotic Dis 19: 152-173.

61. Wickham H, 2016. ggplot2: Elegant Graphics for Data Analysis. New York, NY: Springer-Verlag. 